\title{
High-Speed Railways' Impact on the Connection of Cities along the Line Based on the Analysis of Micro-Blog Data
}

\author{
Chaopeng Li \\ Institute of Regional Development, College of Economics, Jinan University, Guangzhou, China \\ Email: qqq936071372@hotmail.com
}

How to cite this paper: Li, C.P. (2017) High-Speed Railways' Impact on the Connection of Cities along the Line Based on the Analysis of Micro-Blog Data. American Journal of Industrial and Business Management, 7, 566-580.

https://doi.org/10.4236/ajibm.2017.75042

Received: April 6, 2017

Accepted: May 14, 2017

Published: May 17, 2017

Copyright $\odot 2017$ by author and Scientific Research Publishing Inc. This work is licensed under the Creative Commons Attribution International License (CC BY 4.0).

http://creativecommons.org/licenses/by/4.0/

\begin{abstract}
Based on micro-blog big data, this article will conduct an analysis on the follow-up data of every prefecture-level city's micro-blog data and these cities all lie along the Beijing-Guangzhou High-Speed Railway. Then we found that the asymmetry lied in the connection between these cities and there was a provincial-level barrier among them. By comparing the situation before and after the operation of high-speed railway, we realize that this kind of provincial-level barrier still exists. Meanwhile, as the Beijing-Guangzhou High-Speed railway is put into operation and the connection among the cities along the railway has been strengthened, the connection presents the tendency of decentralization.
\end{abstract}

\section{Keywords}

High Speed Railway, Urban Contact, Asymmetry, Provincial-Level Barrier, Decentralization

\section{Introduction}

Urban linkages are one of the important factors influencing the development of a city and urban agglomeration. The state of urban contact is determined by various factors, and traffic is an active factor. China opened the Beijing-Tianjin intercity high-speed rail in 2008, and the high-speed rail significantly enhanced the linkage between the two cities. Since then, the high-speed railway has become a new factor affecting the city's development. From 2008 to 2014, the number of high-speed rail passengers increased from 128 million to 820 million. In 2014, the average daily volume of railway passengers was 6.077 million; the average daily volume of high-speed rail was 2.253 million, accounting for about $37 \%$. In recent years, with the rapid development of high-speed railway in China, the main framework of "four vertical and four horizontal" national high- 
speed rail network is basically formed. Supplemented by extension lines and network lines, high-speed railways basically cover cities which have more than 500,000 people. This marks the arrival of the high-speed rail era. High-speed railway on China's economic and social development has brought a profound impact and changed the domestic socio-economic forms. The open of highspeed rail network makes the relationship between cities more closely, and changes the spatial network structure of the city. So what influence does the development of high-speed railways have on the urban linkages? This is the attention of the majority of scholars. However, the lack of open city passenger and other elements of the flow data, limits the direct observation of urban linkages under the impact of high-speed railway, which is a problem that plagues the related research.

This paper intends to collect large data from social networks and measure the impact of high-speed railways on urban linkages. The main question to be solved in this paper is whether high-speed railways have changed the pattern of urban linkages along the route? If changed, then, what's the direction and the extent of the change? At the same time, this article also attempts to use the social network data with the direction of the property to further analyze urban asymmetry and determine the interaction model between cities. It should be noted that, although the asymmetry of urban contact exists objectively, these public data do not have direction attribute, so urban contact can't be observed and analyzed. While the use of social network data is expected to make a breakthrough in this regard. The analysis of the unidirectional, bi-directional and overall relationship between the characteristics of the relationship between cities or regions to reveal the real linkages, dependencies and impact is very helpful.

\section{Literature Review}

Urban linkages refer to the flow of funds, resources, information, human capital and other factors between cities. The urban network has directional characteristic. The impact of research on urban linkages needs to address two key issues. The first one is the data source; the second one is the calculation method. The academic community has done positive exploration to solve these two issues.

In terms of data, although the flow of goods, goods and services between cities is always taking place which is a direct view of the city's contact, but unfortunately these data are in the hands of different departments, and for various reasons, these data are not announced. In order to break through the limitations of these data which cannot be obtained, the academic community uses the estimated approach. Lifang Xiong et al. constructs a city network system to study the trend of urban contact and urban network evolution through the Baidu index data of city users' attention [1]. Zheng Liu et al. also studied the urban network characteristics of 36 sub-provincial cities through Baidu Index [2]. Feng Zhen et al. analyzed the relationship between cities by analyzing the relationship between friends and the relationship of micro-blog data from the perspective of network society [3]. 
In terms of methods, Bingrong Leng et al. found that urban networks exhibit high clustering coefficients and short path "small world" network characteristics [4]. Pengfei Ni et al. built an interlocking network model with the distribution characteristics of the office network of the productive service enterprises to measure the degree of contact between Chinese cities [5]. Yongling Yao et al. analyzed the concrete manifestations of the differences between cities on the basis of Taylor, and analyzed the different levels of dynamic factors influencing the inter-city contact level [6]. Zhengbing Liu et al. analyzed regional spatial linkages and urban network structures through intercity rail and highway passenger trips [7]. Zhongchen Yang et al. used passenger data to analyze the city's foreign traffic convenience and contact pattern [8].

With the development of the Internet and the availability of network data, some scholars began to study social and economic activities through network data. Dunbar et al. found that the online social network structure can reflect the social network structure in the real world, and they have very similar characteristics [9]. There are many literatures on social network data analysis at home and abroad, such as Twitter and social economy characteristics [10], geographic support and major disaster management [11], geography research based on micro-blog network information [3]. Michael et al. used the Twitter data to analyze the US people's health food and unhealthy food preferences of the regional distribution characteristics [12].

Overall, the study of economic linkages between cities mostly uses the gravitational model which is based on the construction of accessibility. However the gravitational model to measure the degree of economic linkage between cities has its own limitations. The first one is the degree of connection is a potential possibility; and the second one is the gravitational model can't reflect the direction of contact, and one-way association can reveal a deeper link age network between cities. Scholars are eager to solve these two problems in applied research. What's more, most of the existing urban network analysis is a static analysis of network linkages.

The Micro-blog data that reflects the direct contact between cities can be a good solution to solve these two problems. This paper uses Micro-blog data to solve the direction of city's linkage, active and passive issue which is city $i$ is more concerned about city $j$, or City $j$ is more concerned about city $i$, and this can further analyze which ones are "core" in the pattern of mutual attention and which ones are "marginal" in the pattern of mutual attention. The innovation of the research is that we use inter-city Micro-blog data to simulate inter-city linkages and the degree of attention, which can't be solved by using the traditional method. It's rare to use Micro-blog data to analyze the change of city's contact and attention which is caused by the opening of high-speed rail, so we try to do this analysis.

\section{Research Methods}

\subsection{Data Sources and Processing}

Micro-blog as a domestic social networking platform has been widely popular 
since 2010, which allows users to focus reviews and change information on platform. The linkage of point and edge which represents micro-blog users and the number of evaluation and forwards microblog has formed a social network. Based on the huge micro-blog data, we can do a lot of research, such as "small world network" analysis can be studied through the linkage node and chain node, power rate distribution can be studied through the node's entry degree and exit degree, micro-blog user characteristics can be studied through microblog contents and forwards. This paper constructs a social network system by using city's micro-blog two-way frequency which are the prefecture-level city along the high-speed rail, and then study its internal structure and characteristic changes after the opening of high-speed rail.

We can get the dynamic time and space model of city's contact through capturing and analyzing the different periods of micro-blog data. Micro-blog as a dynamic social network platform provides a search page; we can retrieve the number of micro-blog which meets conditions through searching messages such as time range, city's location and keywords, so we prepared a web crawler to simulate the page search process. In addition, Micro-blog provides a search page. The search page not only shows the first 50 pages of the Microblog, but also displays the number of microblogs which meet the conditions in the bottom of the page. According to the time range, the city and keywords, we can get the number of eligible Micro-blog. Through the micro-blog application program interface (API) and the preparation of the reptile program, we obtained a large amount of data from the micro-blog users of the 24 prefecture-level cities along the BeijingGuangzhou high-speed railway, and the data is from January 2010 to November 2014.Through data extraction, we get the number of micro-blog which is about city $i$ refers to city $j$.

\subsection{Urban Contact Measurement Method}

We use social media data to analyze the linkage between cities along the BeijingGuangzhou high-speed rail, the linkage is about the number of micro-blog which is about city $i$ refers to city $j$, The association between such cities is bi-directional asymmetry. The greater the number of microblogs mentioned by a city to another city, the closer the city is to that city. Firstly, through averaging the monthly data for one year as the annual average data to reduce volatility and improve the robustness of the data; secondly, the temporal and spatial volatility of intercity linkage is observed by analyzing the continuity of the monthly data.

This paper uses the analysis of these data to observe the characteristics of the linkage about cities along the Beijing-Guangzhou high-speed railway, and weather the linkage between the cities along the Beijing-Guangzhou high-speed railway has undergone significant changes after the opening of high-speed rail. Then we try to find weather the high-speed rail have had an impact on link ages between the city along the high-speed rail, and what kind of impact. Through the preliminary analysis of the micro-blog data, we find that the linkages between the prefecture-level cities along the Beijing-Guangzhou high-speed rail 
show a typical network structure, and this relationship mainly exist between the provincial cities, non-provincial cities to other province's provincial capital cities, and cities in the same province.

According to the system of prefecture-level cities along high-speed railway, we respectively calculate the two-way association between cities, bidirectional correlation, center degree, inter-city correlation coefficient, network connection degree and urban impact index. This paper uses the following algorithm to measure the characteristics of the prefecture-level city network system along the high-speed rail. That is, using the entry degree, exit degree, the center degree which describes the two-way association between cities to observe the overall situation of a city's external relations; then using standardized data to observe the distribution of a city's attention on other cities' along the high-speed rail. The software used in the empirical analysis is MATLAB R2013a.

\subsubsection{The Two-Way Association between Cities}

In the micro-blog data, $P_{i j}$ is the number of micro-blog which is about city $i$ referring to city $j$, that is city $i$ s attention on city $j, P_{j i}$ is the number of micro-blog which is about city $j$ referring to city $i$, that is city $j$ s attention on city $i . P_{i j}$ represents the directional connection between city $i$ and city $j$ and the values of them are different; each city as a node, the directional connection between cities as a weighted side, so that it constitutes a social network. In the social network, entry degree and exit degree are important indicators to measure node characteristics.

We define $A$ as entry degree, which is about other cities' attention on city $i$, and the formula is as follows:

$$
A_{i}=\frac{\sum_{j} P_{i j}}{\sum_{i} \sum_{j} P_{i j}}(i \neq j)
$$

Similarly, we define $B$ as exit degree, which is about city is attention on other cities, and the formula is as follows:

$$
B_{i}=\frac{\sum_{j} P_{j i}}{\sum_{i} \sum_{j} P_{i j}}(i \neq j)
$$

\subsubsection{Center Degree}

The center degree reflects the status of a single city in the whole city network, the right of ownership, and the position of the occupied position. We define $\mathrm{L}$ as the center degree, and we use the harmonic average to calculate entry degree and exit degree to evaluate the center degree of one city. The formula is as follows:

$$
L_{i}=\frac{2 A_{i} B_{i}}{A_{i}+B_{i}}(i \neq j)
$$

\subsubsection{Inter-City Correlation Coefficient}

The correlation coefficient between cities is measured from the whole, and the degree of attention is different. The correlation coefficient between cities shows the degree of the association between the cities. 
We use the following algorithm to express the directional strength between the two:

$$
C_{i j}=\frac{p_{i j}}{\sum_{j} P_{i j}}(i \neq j)
$$

Since the city $i$ and the city $j$ are bidirectional, we use the following algorithm to express the difference between the two interrelated correlation coefficient:

$$
D_{i j}=C_{i j} * C_{j i}
$$

\subsubsection{Network Connectivity}

Let $\operatorname{Max}\left(C_{i j}\right)=100$, use the maximum standardization:

$$
D_{i j}^{\prime}=\frac{D_{i j}}{\operatorname{Max}\left(D_{i j}\right)} * 100
$$

$D_{i j}^{\prime}$ is the maximum value of the interconnected degree between city $i$ and $j$.

$$
E_{i}=\Sigma D_{i j}^{\prime}(i \neq j)
$$

$E_{i}$ is the network connectivity of city $i$.

\subsubsection{Urban Impact Index}

The city impact index indicates the ability of a city to influence other cities, defined as:

$$
F_{i}=\frac{\sum_{i} C_{j i}}{n-1}(i \neq j)
$$

$F_{i}$ represents the influence of the city $i$, representing the degree of dependence of other cities on city $i$.

\section{Result Analysis}

In the analysis of the results, firstly we observe whether the inter-city linkage has changed after the opening of the high-speed railway; secondly we analyze the performance of the change at the spatial level, thirdly we analyze the change in the time series. We use the analysis of these three aspects to find the characteristics of urban linkages. We distinguish five phenomena as follows: (1) a city not only attentions about other cities but also is concerned by other cities; (2) the overall change in the degree of attention among all the cities; (3) the degree of attention between cities after the opening of high-speed railway; (4) whether the degree of attention between cities is related to the level of the city; (5) whether the degree of attention is related to the spatial distance between each other.

\subsection{Analysis of the Differences in the Two-Way Relationship between Regions}

Through the analysis of Table 1, we found that along the Beijing-Guangzhou high-speed rail, Beijing's exit degree increased significantly, Zhengzhou's exit degree increased slightly; Guangzhou, Wuhan, Changsha, Shijiazhuang's exit degree significantly decreased; other cities did not change significantly. Beijing, 
Table 1. City's entry degree, exit degree and center degree.

\begin{tabular}{|c|c|c|c|c|c|c|}
\hline \multirow{2}{*}{ Year } & \multicolumn{2}{|c|}{ A } & \multicolumn{2}{|c|}{ B } & \multicolumn{2}{|c|}{$\mathrm{L}$} \\
\hline & 2010 & 2014 & 2010 & 2014 & 2010 & 2014 \\
\hline Beijing & 0.274 & 0.608 & 0.319 & 0.208 & 0.295 & 0.310 \\
\hline Baoding & 0.007 & 0.006 & 0.011 & 0.014 & 0.008 & 0.009 \\
\hline Shijiazhuang & 0.041 & 0.024 & 0.014 & 0.028 & 0.021 & 0.026 \\
\hline Xingtai & 0.002 & 0.005 & 0.003 & 0.007 & 0.003 & 0.005 \\
\hline Handan & 0.003 & 0.004 & 0.006 & 0.011 & 0.004 & 0.006 \\
\hline Anyang & 0.002 & 0.005 & 0.005 & 0.008 & 0.002 & 0.006 \\
\hline Hebi & 0.000 & 0.001 & 0.001 & 0.002 & 0.000 & 0.001 \\
\hline Xinxiang & 0.002 & 0.004 & 0.004 & 0.007 & 0.003 & 0.005 \\
\hline Zhengzhou & 0.054 & 0.057 & 0.027 & 0.063 & 0.036 & 0.060 \\
\hline Xuchang & 0.002 & 0.003 & 0.002 & 0.004 & 0.002 & 0.003 \\
\hline Luohe & 0.001 & 0.002 & 0.001 & 0.003 & 0.001 & 0.002 \\
\hline Zhumadian & 0.001 & 0.001 & 0.002 & 0.004 & 0.001 & 0.002 \\
\hline Xinyang & 0.001 & 0.003 & 0.005 & 0.007 & 0.002 & 0.004 \\
\hline Xiaogan & 0.001 & 0.007 & 0.002 & 0.003 & 0.002 & 0.004 \\
\hline Wuhan & 0.058 & 0.033 & 0.080 & 0.110 & 0.067 & 0.051 \\
\hline Xianning & 0.001 & 0.002 & 0.002 & 0.003 & 0.001 & 0.002 \\
\hline Yueyang & 0.002 & 0.004 & 0.005 & 0.005 & 0.003 & 0.004 \\
\hline Changsha & 0.051 & 0.026 & 0.052 & 0.071 & 0.051 & 0.038 \\
\hline Zhuzhou & 0.003 & 0.003 & 0.005 & 0.007 & 0.004 & 0.004 \\
\hline Hengyang & 0.004 & 0.003 & 0.006 & 0.006 & 0.004 & 0.004 \\
\hline Chenzhou & 0.005 & 0.001 & 0.004 & 0.003 & 0.004 & 0.004 \\
\hline Shaoguan & 0.006 & 0.004 & 0.023 & 0.007 & 0.010 & 0.005 \\
\hline Guangzhou & 0.352 & 0.094 & 0.198 & 0.227 & 0.254 & 0.133 \\
\hline Shenzhen & 0.128 & 0.100 & 0.223 & 0.191 & 0.163 & 0.132 \\
\hline
\end{tabular}

Shenzhen, Shaoguan's entry degree declined, Guangzhou, Zhengzhou, Wuhan, Shijiazhuang, Changsha's entry degree increased significantly, the extent of other urban changes are not obvious.

Through the analysis of 2014 entry degree and exit degree, we found that along the Beijing-Guangzhou high-speed rail, Beijing's exit degree is far greater than entry degree, Guangzhou, Wuhan, Shenzhen, Changsha's entry degree is significantly greater than exit degree, the other cities do not have obvious difference between their entry degree and exit degree. This shows that the provincial capital and first-tier cities have larger difference between entry degree and exit degree. The provincial capital and first-tier cities' entry degree is significantly greater than exit degree, mainly due to the provincial capital and first-tier cities are the core in political, economic and population. 


\subsection{Center Degree Analysis}

The center degree which refers to the core degree of a node at the network system in Table 1 reflects the degree of the node city's attention on other cities and the ability to be cared by other cities. Through the analysis of Chartone, we find that the center degree of Guangzhou, Wuhan, Shenzhen, Changsha and Shaoguan has decreased significantly in the cities along the Beijing-Guangzhou highspeed railway, and the rest cities' center degree has increased. This indicates that with the opening of high-speed railway, non-provincial prefecture-level cities' core status has increased in the system of the cities along the Beijing-Guangzhou high-speed railway, and high-speed railway has strengthened the interaction of information and resources flow with other cities. Although the center degree of most capital cities and other major cities has declined, the core status of the capital cities and first-tier cities is still solid in the whole network system, which is because the capital cities and first-tier cities occupy a relatively large number of resources, and have better health education and cultural facilities and the environment.

The total center degree refers to the central degree of the whole network system, and reflects the overall concentration of the whole network system. The centralization trend of the whole system can be found through the time variation of the total center system. The total city center degree of the prefecture-level cities along the Beijing-Guangzhou high-speed rail has reduced from 0.941 to 0.819 since 2010 to 2014 . From the analysis of the total center degree's change, the whole network system has presented the characteristics of decentralization. This shows that the opening of high-speed rail enhances the linkages of cities along the high-speed rail, and enhances the flow of resources. The entire network system presents decentralization characteristics.

\subsection{Interregional Correlation Coefficient Analysis}

From the comparison of Figure 1 and Figure 2, we can see that the attention among the cities are unequal, such as other cities' one-way attention degree on Beijing is relatively high, and Beijing's one-way attention degree on other cities is relatively weak in spite of Shijiazhuang, Zhengzhou, Wuhan, Changsha, Guangzhou, Shenzhen. By comparing the city's directional and non-directional graph from 2010 to 2014, we can find that other cities' one-way attention degree on Beijing has increased in spite of Shijiazhuang and Hebe.

From the comparison of Figure 3 and Figure 4, we find that there are provincial barriers between city linkages, and the contact of non-provincial capitals is limited within the province. The contact between adjacent non-provincial provinces of non-capital cities is weak. Through comparing the figure of 2010 and 2014, we find that the opening of the high-speed railway hasn't broken the provincial barriers.

\subsection{Network Connectivity Analysis}

We analyze the city's network connectivity through the analysis of attention de- 


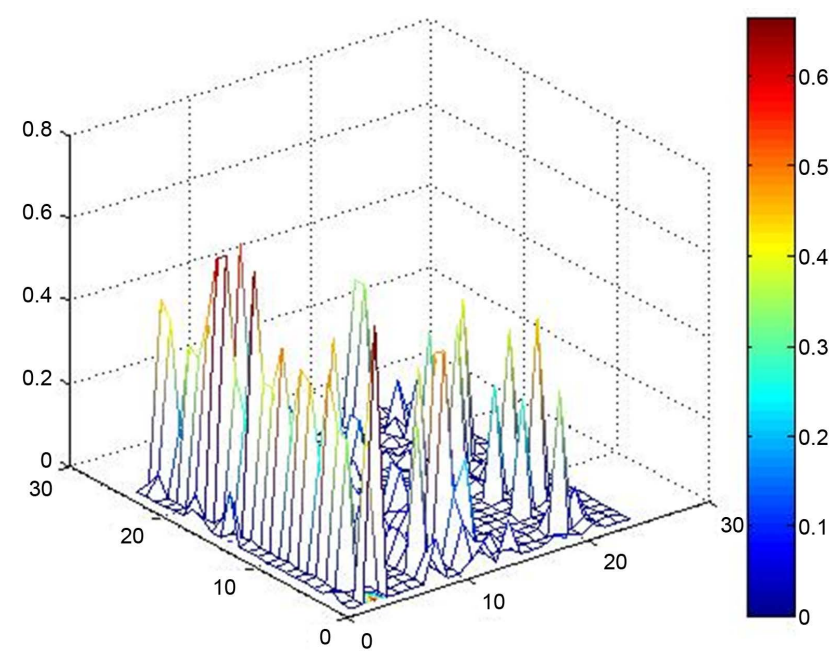

Figure 1. The graph of city's directional attention in 2010, drawn by MATLAB R2013a.

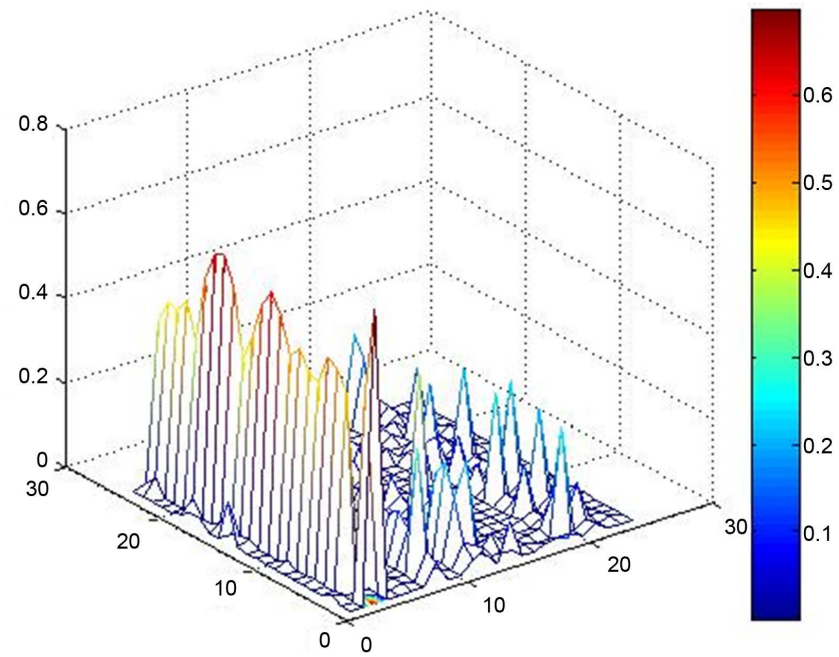

Figure 2. The graph of city's directional attention in 2014, drawn by MATLAB R2013a.



Figure 3. The graph of city's nondirectional attention in 2010, drawn by MATLAB R2013a. 


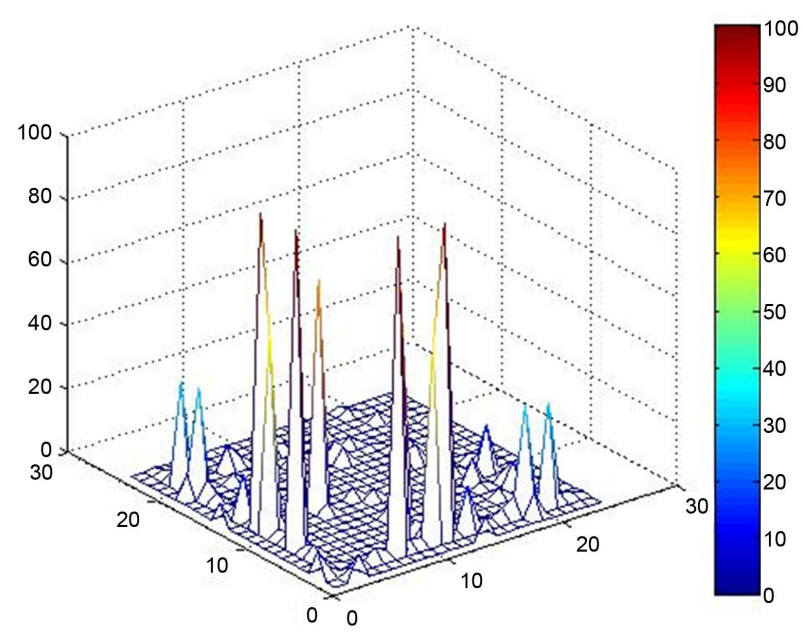

Figure 4. The graph of city's nondirectional attention in 2014, drawn by MATLAB R2013a.

gree about the flow of standardized data 2. According to the comparative analysis in Table 2, we find that there are obvious differences in the degree of the network connection between cities. Beijing, Guangzhou and Shenzhen are the first grade, and their contact degree is above 150; Zhengzhou, Wuhan and Changsha are the second grade, and their contact degree is between 50 and 150; Baoding, Shijiazhuang, Xingtai, Anyang, Hebi, Xinxiang, Xuchang, Luohe, Zhumadian, Xinyang, Xiaogan, Xianning, Yueyang, Zhuzhou, Hengyang, Chenzhou, Shaoguan are the third level, and their contact degree is between 0 and 50. From the Chart two we can see that the degree of the linkage between the non-provincial cities is far lower than that of the capital cities, and the degree of the linkage between the non-provincial cities is not very different.

By comparing the degree of contact between 2010 and 2014, we find that, in spite of Beijing, Guangzhou, Shenzhen, the contact degree of other cities does not change significantly. Beijing's network connectivity has increased from 247.6 to 380.7; Shenzhen's contact degree has increased from 159.8 to 180.5 ; and Guangzhou's contact degree has declined slightly from 220.4 to 187 . The total network connectivity along the prefecture-level cities along the high-speed rail has increased from 997.83 in 2010 to 1138.86 in 2014, and it is found that the opening of the high-speed rail has significantly strengthened the degree of the inter-city linkage.

\subsection{Urban Impact Index Analysis}

The urban impact index reflects the size of the city's overall impact on other cities. According to the Urban impact index of Table 2, it can be found that the distribution of urban impact index between cities shows a significant stratification phenomenon, the first level is Beijing, the urban impact index is above 9; the second grade is Zhengzhou, Wuhan, Changsha, Guangzhou and Shenzhen, the degree of their urban impact index is between 1 and 4; the third level is other cities, the degree of urban impact index is between 0 and 1 . Based on the com- 
Table 2. City's network connectivity and urban impact index.

\begin{tabular}{|c|c|c|c|c|}
\hline \multirow{2}{*}{ Year } & \multicolumn{2}{|c|}{ E } & \multicolumn{2}{|c|}{$\mathrm{F}$} \\
\hline & 2010 & 2014 & 2010 & 2014 \\
\hline Beijing & 247.571 & 380.685 & 9.684 & 11.688 \\
\hline Baoding & 13.209 & 16.000 & 0.224 & 0.237 \\
\hline Shijiazhuang & 28.085 & 31.812 & 0.470 & 0.411 \\
\hline Xingtai & 5.212 & 8.703 & 0.079 & 0.158 \\
\hline Handan & 8.930 & 12.532 & 0.147 & 0.209 \\
\hline Anyang & 9.064 & 7.103 & 0.168 & 0.190 \\
\hline Hebi & 1.578 & 2.367 & 0.017 & 0.043 \\
\hline Xinxiang & 8.811 & 11.136 & 0.111 & 0.200 \\
\hline Zhengzhou & 54.837 & 63.503 & 2.488 & 1.716 \\
\hline Xuchang & 5.731 & 4.733 & 0.077 & 0.093 \\
\hline Luohe & 4.335 & 2.830 & 0.051 & 0.086 \\
\hline Zhumadian & 4.365 & 4.279 & 0.053 & 0.084 \\
\hline Xinyang & 10.609 & 10.462 & 0.167 & 0.194 \\
\hline Xiaogan & 5.261 & 4.761 & 0.029 & 0.060 \\
\hline Wuhan & 70.380 & 82.797 & 2.052 & 1.491 \\
\hline Xianning & 7.820 & 5.190 & 0.032 & 0.076 \\
\hline Yueyang & 12.077 & 9.882 & 0.102 & 0.135 \\
\hline Changsha & 67.576 & 73.221 & 1.907 & 1.456 \\
\hline Zhuzhou & 13.759 & 17.185 & 0.094 & 0.144 \\
\hline Hengyang & 14.724 & 9.995 & 0.108 & 0.140 \\
\hline Chenzhou & 4.136 & 4.545 & 0.082 & 0.072 \\
\hline Shaoguan & 19.571 & 7.618 & 0.100 & 0.104 \\
\hline Guangzhou & 220.381 & 186.998 & 3.633 & 2.684 \\
\hline Shenzhen & 159.804 & 180.525 & 2.128 & 2.330 \\
\hline
\end{tabular}

parative analysis of the influence of Beijing-Guangzhou high-speed rail along the 2010 and 2014, we find that the urban impact index has increased in spite of Zhengzhou, Wuhan, Changsha and Guangzhou, and the increase is the most obvious in Beijing.

\subsection{Space-Time Analysis}

Beijing, Guangzhou, Shenzhen is the national first-tier cities, Zhengzhou is the Central Plains economic group, Wuhan is the middle reaches of the Yangtze River economic group of the core cities, so we choose this five representative cities to study city's network connectivity and urban impact index. BeijingGuangzhou high-speed railway was opened in December 2012.According to the 


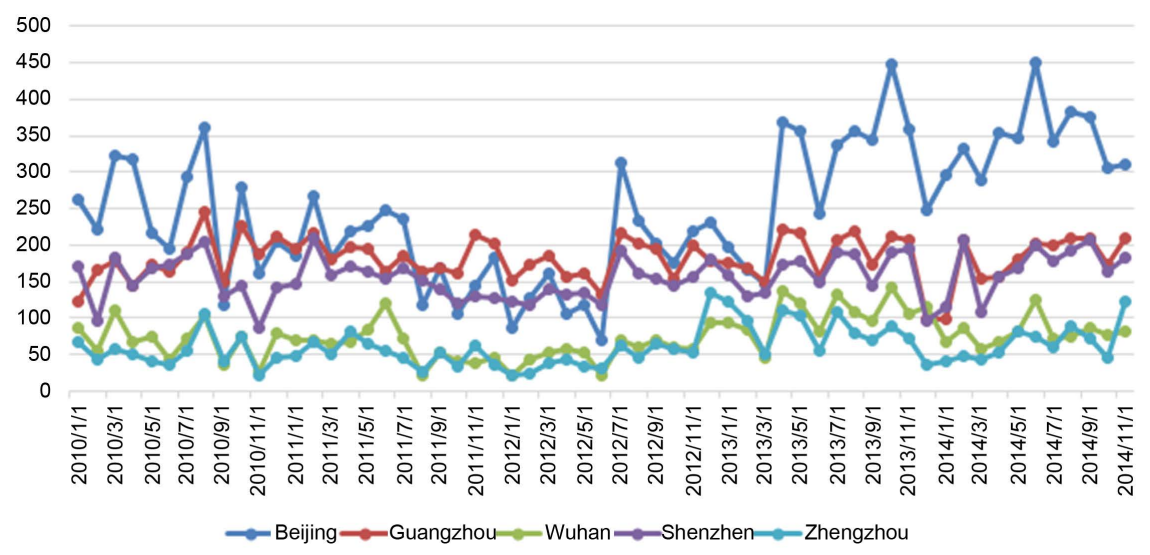

Figure 5. Network connectivity.

comparative analysis in Figure 5, we find that Beijing's network connectivity had increased significantly after the opening of Beijing-Guangzhou high-speed railway and expanded the gap with Guangzhou and Shenzhen. Guangzhou, Shenzhen's increase is not obvious, but its fluctuation characteristics are obvious. Zhengzhou, Wuhan's network connectivity rises slightly in spite of some month. Network connectivity is affected by unexpected events, and the network connectivity between different months fluctuates greatly.

Through the comparative analysis of the urban impact index in Figure 6, we find that the urban impact index of Beijing is much higher than that of Guangzhou, Shenzhen, Zhengzhou and Wuhan, and the influence degree of Beijing is increased obviously after the opening of high-speed railway. The impact of Guangzhou, Shenzhen, Zhengzhou, Wuhan has not significantly layer, but the impact of Guangzhou has always been slightly higher than the other three cities.

On the whole, with the opening of the Beijing-Guangzhou high-speed railway, Beijing as the country's political, economic and cultural center, its status has been enhanced, and gradually expanded the gap with other cities. Although Guangzhou, Shenzhen's current network connectivity and urban impact index are higher than Zhengzhou, Wuhan, but this gap has a narrowed trend.

Figure 7 shows Beijing's attention degree on Guangzhou, Shenzhen, Zhengzhou, Wuhan and Guangzhou, Shenzhen, Zhengzhou, Wuhan's attention degree on Beijing. The data is from January 2010 to November 2014 which has 59 consecutive months.

Figure 7 is a good description of the difference between the inter-city linkages. We found that Guangzhou, Shenzhen, Zhengzhou, Wuhan's attention degree on Beijing is clearly stronger than Beijing's attention degree on Guangzhou, Wuhan, Shenzhen, Zhengzhou. Beijing as the center of national political, economic and cultural, it is inevitable to have such result. Beijing's attention degree on other cities and other cities' attention degree on Beijing have a rising trend, and other cities attention on Beijing's fluctuates strongly. At the same time, Beijing's attention on Guangzhou, Shenzhen, Zhengzhou and Wuhan is stratified, while Guangzhou, Shenzhen, Zhengzhou and Wuhan's attention on Beijing have no signs of stratification. 


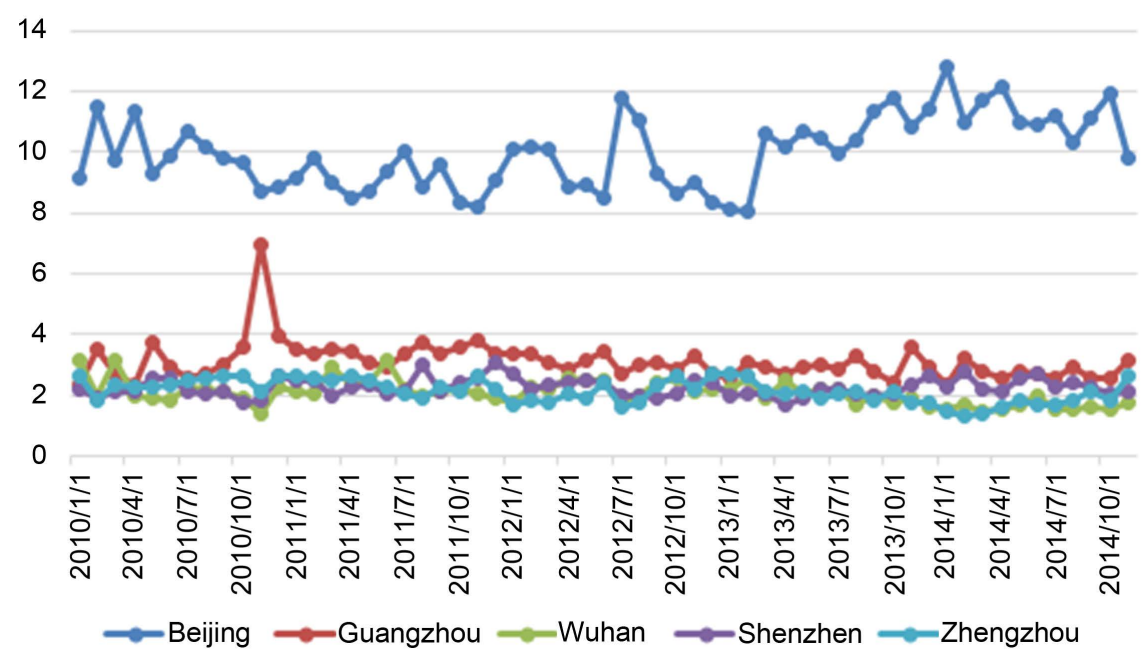

Figure 6. Urban impact index.

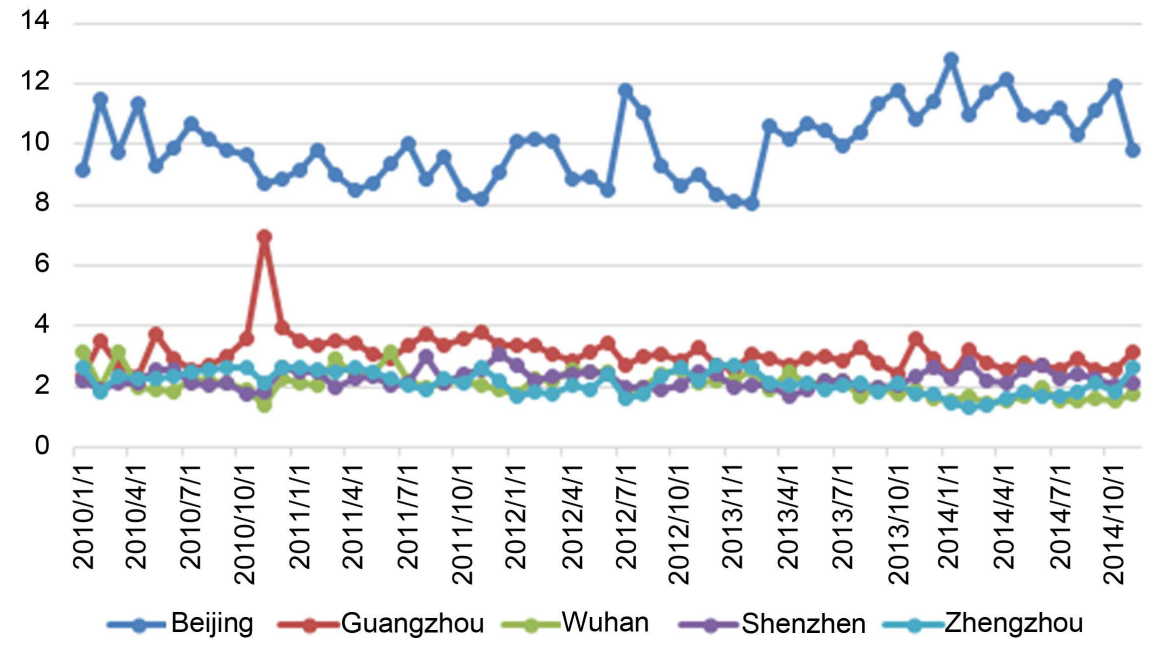

Figure 7. Beijing's attention on Guangzhou, Wuhan, Shenzhen, Zhengzhou and Guangzhou, Wuhan, Shenzhen, Zhengzhou's attention on Beijing.

\section{Conclusions and Discussion}

Micro-blog data has the characteristics of time and space, and can measure the network connection characteristics between cities according to different spatial scales and time intervals, so we can measure and forecast the relationship between cities or regions in multi-scale.

The innovation of the article is that we analyze the high-speed railway's effect on the cities' linkage along the Beijing-Guangzhou High-Speed railway by using micro-blog data. We mainly solve two problems in the analysis of urban linkage which exist in the literature of other scholars, the first one is that the degree of city's connection is a potential possibility; and the second one is that the conventional Gravitational Model can't reflect the direction of contact. Besides, we make further efforts to do dynamic analysis of urban network linkages. This paper uses micro-blog data to solve the direction of city's linkage, active and passive issue which is city $i$ is more concerned about city $j$, or city $j$ is more con- 
cerned about city $i$, and this can further analyze which one is "core" in the pattern of mutual attention and which one is "marginal" in the pattern of mutual attention. The next innovation of the research is that we use inter-city micro-blog data to simulate inter-city linkages and the degree of attention, which can't be solved by using the traditional method. It's rare to use micro-blog data to analyze the change of city's contact and attention which is caused by the opening of high-speed rail.

The main contribution of this research: In this paper, a new method based on the location data and the information of the place names in the social media is used to evaluate the relationship between cities. In view of the problem that the relationship between cities is not symmetrical, this method can provide the ability to evaluate the relationship between two cities, and provide a new method to measure the influence and similarity of cities. The problem that the relationship between cities is not symmetrical has been realized in the study of economic geography, regional economy and spatial economy, but they suffer from the problem that there is no suitable method to solve it. Therefore, it is very helpful for us to reveal the relationship between the cities. The analysis of a particular event can also be done using these methods, such as the fire explosion in Tianjin. Because social media data has temporal and spatial characteristics of natura, urban linkages can be measured separately according to the size and characteristics of the city between the different time intervals, thus provide a measure and forecast a multi-scale regional relationship means. Finally, due to the temporal attributes of social media, it can provide the evaluation ability of different time scales, and provide the ability of time based retrieval.

Through the static analysis, we find the asymmetry of the inter-city linkages and through the analysis of the data, the degree of the relationship between the cities is measured. At the same time, the degree of the urban network system along the high-speed railway is analyzed: center degree and influence degree, and reveals the spatial structure of urban network system along high speed railway. Through the dynamic comparison and analysis, the influence of high-speed railway opening on the urban network is studied. Through the analysis of time series, this paper studies the changing characteristics of Beijing's connection to Guangzhou, Shenzhen, Zhengzhou, Wuhan and Guangzhou, Shenzhen, Zhengzhou, Wuhan's connection to Beijing.

Based on the analysis of the micro-blog data of cities along the BeijingGuangzhou high-speed railway, the results show that the inter-city linkages exist mainly between the provincial capitals, the inter-city cities and the provincial capital cities. And this kind of connection shows obvious asymmetry. Beijing, Guangzhou and other big cities and capital cities have the advantages of economy, education and population; these cities are paid much more attention than other cities, and their degree of influence is far greater than other cities. In addition, it is found that there are provincial barriers in the inter-city linkages, and the inter-linkages between non-provincial cities are confined to their own provinces. The neighboring non-provincial provinces are weakly linked. By the anal- 
ysis of the high-speed rail's influences, we find that the barrier dose not disappear due to the opening of the high-speed rail. At the same time, the opening of the Beijing-Guangzhou high-speed railways has strengthened the flow of resources and elements; the linkage between the cities along the high-speed rail has showed a center-oriented feature.

\section{References}

[1] Xiong, L.F., Zhen, F., Xi, G.L., et al. (2014) Characteristics of Urban Network Change in China's Three Major Economic Zones-An Empirical Study Based on Baidu's Information Flow. Tropical Geography, 1, 34-43.

[2] Liu, Z., Wang, S.F., Zhao, M.X., et al. (2013) Exploratory Analysis of Directed Weighted Urban Networks. Geography Research, 7, 1253-1268.

[3] Zhen, F., Wang, B. and Chen, Y.X. (2012) Chinese Urban Network Characteristics Based on Network Social Space-Taking Sina Weibo as an Example. Acta Geographica Sinica, 8, 1031-1043.

[4] Leng, B.-R., Yang, Y.-C., Li, Y.-J. and Zhao, S.-D. (2011) Spatial Characteristics and Complexity Analysis of Urban Economic Network Structure in China. Acta Geographica Sinica, 2, 199-211.

[5] Ni, P.F., Liu, K. and Taylor, P.J. (2011) China's Urban Linkageages: Based on the Interlocking Network Model. Comparison of Economic and Social Systems, 6, 96103.

[6] Yao, Y.-L., Dong, Y. and Wang, Y.-H. (2012) Comparison of Energy Levels and Dynamic Factors of Global Urban Networks in Beijing and Seoul. Economic Geography, 8, 37-42.

[7] Liu, Z.-B., Liu, J.-Y., He, X.-P. and Wang, F.-Z. (2014) Analysis of Urban Spatial Connection and Its Network Pattern in Zhongyuan Economic Zone-Based on Intercity Passenger Flow. Public Geography, 7, 58-66.

[8] Yang, Z.-C. and Lu, Y.-Q. (2014) Study on the Convenience and Connection Pattern of Urban Traffic in Shandong Province Based on Passenger Flow. Polonomic Geography, 9, 63-67.

[9] Dunbar, R.I.M., Arnaboldi, V., Conti, M., et al. (2015) The Structure of Online Social Networks Mirrors Those in the Offline World. Social Networks, 10, 39-47.

[10] Alampay, E.A. (2006) Analyzing Socio-Demographic Differences in the Access \& Use of ICTs in the Philippines Using the Capability Approach. Ejisdc the Electronic Journal on Information Systems in Developing Countries, 27, 1-39.

[11] Michael, F. and Goodchild, J. (2010) Crowdsourcing Geographic Information for Disaster Response: A Research Frontier. International Journal of Digital Earth, 3, 231-241. https://doi.org/10.1080/17538941003759255

[12] Widener, M.J. and Li, W. (2014) Using Geolocated Twitter Data to Monitor the Prevalence of Healthy and Unhealthy Food References across the US. Applied Geography, 10, 189-197. 
Submit or recommend next manuscript to SCIRP and we will provide best service for you:

Accepting pre-submission inquiries through Email, Facebook, LinkedIn, Twitter, etc. A wide selection of journals (inclusive of 9 subjects, more than 200 journals)

Providing 24-hour high-quality service

User-friendly online submission system

Fair and swift peer-review system

Efficient typesetting and proofreading procedure

Display of the result of downloads and visits, as well as the number of cited articles Maximum dissemination of your research work

Submit your manuscript at: http://papersubmission.scirp.org/

Or contact ajibm@scirp.org 